\title{
Fibrosarcomatous pigmented dermatofibrosarcoma protuberans: A case report with review of the literature
}

\author{
MITSUAKI ISHIDA and HIDETOSHI OKABE \\ Department of Clinical Laboratory Medicine and Division of Diagnostic Pathology, \\ Shiga University of Medical Science, Otsu, Shiga 520-2192, Japan \\ Received February 22, 2012; Accepted June 12, 2012
}

DOI: $10.3892 / \mathrm{ol} .2012 .765$

\begin{abstract}
Dermatofibrosarcoma protuberans (DFSP) is a relatively rare low-grade sarcoma that occasionally exhibits melanin-containing spindle cells within the tumor. Fibrosarcomatous DFSP (FS-DFSP) is a variant that is characterized by areas which are histopathologically indistinguishable from fibrosarcoma. In the present study, we describe a case of fibrosarcomatous pigmented DFSP and review the clinicopathological features of this extremely rare lesion. A 51-year-old male presented with a slow-growing nodular cutaneous mass in his left upper arm. Histopathologically, the resected tumor was comprised of pigmented DFSP in approximately $20 \%$ of the tumor, with the remaining area further covered by a fibrosarcomatous component. A review of the clinicopathological features of the five previously reported cases as well as the present case indicated that this lesion mainly affects middle-aged males and occurs mostly in the extremities. Melanin-containing spindle cells are present only in the conventional DFSP component. The prognosis appears to be poor; in the six cases reviewed, four demonstrated multiple metastases and three succumbed to the disease. Our analyses revealed that the presence of a fibrosarcomatous component in pigmented DFSP is associated with aggressive behavior; therefore, careful assessment for the presence of a fibrosarcomatous component is necessary in the diagnosis of this disease.
\end{abstract}

\section{Introduction}

Dermatofibrosarcoma protuberans (DFSP) is a relatively rare distinct dermal and subcutaneous neoplasm, which is generally regarded as a low-grade sarcoma (1). Although DFSP is

Correspondence to: Dr Mitsuaki Ishida, Department of Clinical Laboratory Medicine and Division of Diagnostic Pathology, Shiga University of Medical Science, Tsukinowa-cho, Seta, Otsu, Shiga 520-2192, Japan

E-mail: mitsuaki@belle.shiga-med.ac.jp

Key words: dermatofibrosarcoma protuberans, fibrosarcoma, Bednar tumor characterized clinically as locally aggressive with a high rate of local recurrence, distant metastases are rare. Several histopathological variants of DFSP, including pigmented DFSP and fibrosarcomatous DFSP, have been reported. Pigmented DFSP, also known as Bednar tumor, is characterized by the presence of melanin-containing dendritic cells within the tumor. The incidence of this variant is quite low, accounting for less than 5\% of all DFSP cases (2). Fibrosarcomatous DFSP (FS-DFSP) is a further variant, characterized by areas histopathologically indistinguishable from fibrosarcoma, and with a clinically increased incidence of metastasis (3). Fibrosarcomatous pigmented DFSP is extremely rare; only five cases have been reported in the English language literature (3-7). In the present study, we report a further case of this extremely rare lesion and discuss the clinicopathological features.

\section{Patients and methods}

Case report. A 51-year-old Japanese man presented with a slow-growing nodular cutaneous mass in his left upper arm, which was first detected two months earlier. The tumor was reddish to purplish in color and measured $4 \times 4 \mathrm{~cm}$ in size. Assessment of the biopsy specimen indicated DFSP; thus, total resection of the tumor with 3-cm margins was performed. This study was approved by the Ethics Committee of Shiga University of Medical Science. Written informed consent was obtained from the patient prior to the study.

The postoperative course was uneventful, and no recurrence or metastasis was observed during 2 months of medical follow-up.

Materials. The formalin-fixed, paraffin-embedded tissue blocks of the resected tumor were cut into 3-micrometerthick sections, deparaffinized and rehydrated. Each section was stained with hematoxylin and eosin (H\&E) and used for immunostaining. Immunohistochemical analyses were performed using an autostainer (Benchmark XT, Ventana Medical Systems, Tucson, AZ, USA) according to the manufacturer's instructions. The following primary antibodies were used: a mouse monoclonal antibody against CD34 (QBEnd/10, Novocastra Laboratories, Ltd., Newcastle upon Tyne, UK) and a mouse monoclonal antibody against p53 protein (PAb1801, Novocastra). 

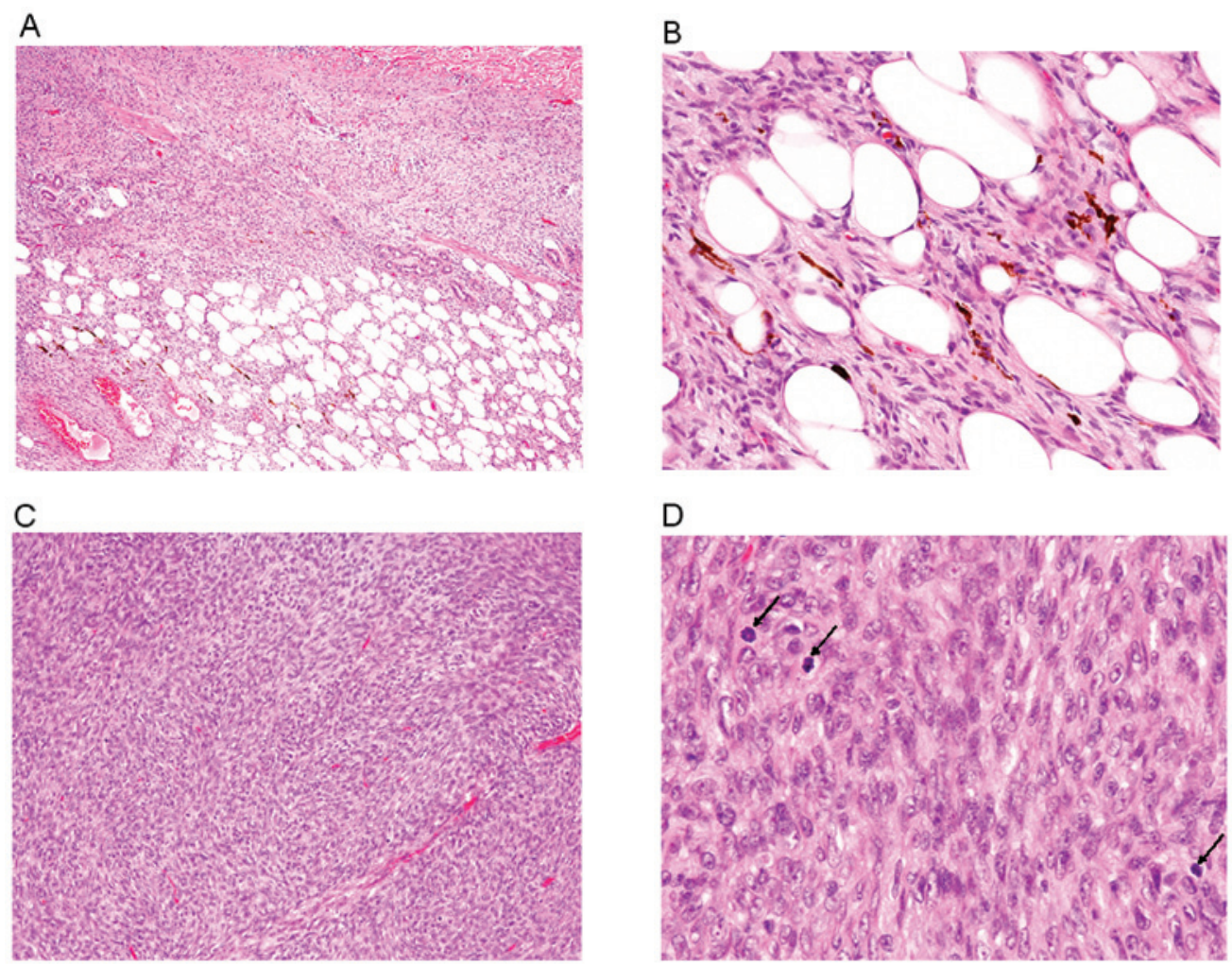

Figure 1. Histopathological findings. (A) Spindle cell proliferation in the dermis with invasion into the subcutis accompanied by honeycomb pattern. Melanincontaining dendritic cells are also observed within the lesion (H\&E staining; original magnification, $\mathrm{x} 40$ ). (B) Spindle cells have mildly enlarged slender nuclei and inconspicuous nucleoli, intermingled with melanin-containing spindle cells. Mitotic figures are rare (H\&E staining; original magnification, x200). (C) More cellular proliferation of atypical spindle cells with herringbone appearance (H\&E staining; original magnification, $\mathrm{x} 40$ ). (D) Spindle cells have large atypical nuclei with conspicuous nucleoli, and mitotic figures are scattered (arrows) (H\&E staining; original magnification, x200).
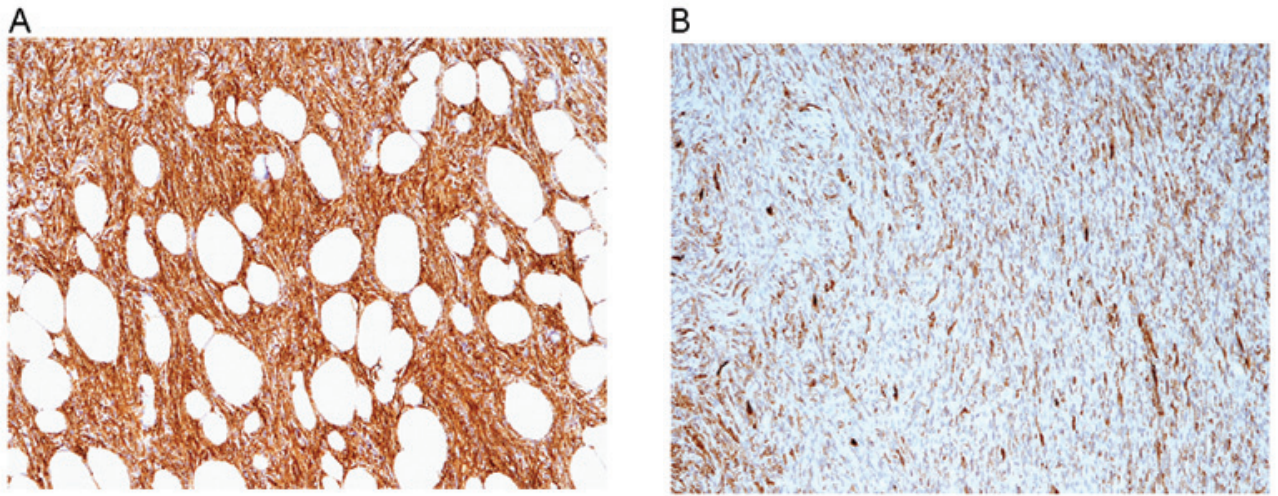

Figure 2. Immunohistochemical findings. (A) CD34 is diffusely and strongly expressed in the pigmented dermatofibrosarcoma protuberans component (original magnification, x100). (B) CD34 is focally expressed in the fibrosarcomatous component (original magnification, x100).

\section{Results}

Histopathological study of the resected tumor revealed monotonous proliferation of uniform spindle cells with mildly enlarged slender nuclei and inconspicuous nucleoli in the dermis and subcutis with storiform appearance (Fig. 1A and B). The epidermis was not involved; however, these spindle cells invaded into the deep subcutaneous tissue with a characteristic honeycomb pattern. Mitotic figures were occasionally observed (1/10 high-power fields). In certain areas, melanin-containing spindle cells, which were bleached by the peroxide method, were scattered. Approximately $20 \%$ of the tumor contained the above-mentioned typical pigmented DFSP (Bednar tumor), and in addition, further cellular spindle cell proliferation was clearly visible. The spindle cells were arranged in fascicles with a herringbone appearance (Fig. 1C) and had large round to short spindle-shaped nuclei with a conspicuous nucleolus (Fig. 1D). Mitotic figures were frequently observed in this region (23/10 high-power fields). These histopathological features resembled fibrosarcoma, and no melanin-containing spindle cells were present in this area. Moreover, no pleomorphic sarcoma components or necrosis were observed. 
Table I. Clinicopathological features of fibrosarcomatous pigmented dermatofibrosarcoma protuberans.

\begin{tabular}{|c|c|c|c|c|c|}
\hline Case no. & $\begin{array}{l}\text { Age/ } \\
\text { gender }\end{array}$ & Location & $\begin{array}{l}\text { Fibrosarcomatous } \\
\text { component } \\
\text { in primary lesion }\end{array}$ & Prognosis & Ref. \\
\hline 1 & $45 / \mathrm{M}$ & Upper arm & - & $\begin{array}{l}\text { Multiple metastases in skin, lung, brain, thyroid, } \\
\text { pancreas, stomach and small intestine. DOD. }\end{array}$ & 4 \\
\hline 2 & $75 / \mathrm{M}$ & Thigh & NA & Metastases in lung and bone. DOD. & 3 \\
\hline 3 & $46 / \mathrm{F}$ & Foot & - & Local recurrence & 5 \\
\hline 4 & $51 / \mathrm{M}$ & Shoulder & - & Metastases in lung, retroperitoneum and colon. DOD. & 6 \\
\hline 5 & $27 / \mathrm{M}$ & Wrist & + & $\begin{array}{l}\text { Multiple metastases in thigh, chest wall, lung, } \\
\text { supraclavicular and parapharyngeal regions }\end{array}$ & 7 \\
\hline Present case & $51 / \mathrm{M}$ & Upper arm & + & No recurrence or metastasis 2 months after surgery & \\
\hline
\end{tabular}

DOD, died of disease; F, female; M, male; NA, not available.

Immunohistochemically, the spindle cells in the pigmented DFSP area were diffusely and strongly positive for CD34 (Fig. 2A), whereas those of the fibrosarcomatous area were focally and weakly positive for this marker (Fig. 2B). Overexpression of $\mathrm{p} 53$ protein was not observed in either the pigmented DFSP or the fibrosarcomatous components.

According to these findings, an ultimate diagnosis of fibrosarcomatous pigmented DFSP (Bednar tumor) was made.

\section{Discussion}

FS-DFSP is a relatively rare variant of DFSP, accounting for approximately $10 \%$ of cases (3). Although conventional DFSP has a significant risk of local recurrence (20-50\%), distant metastasis is rare $(<5 \%)(1,8)$. The local recurrence rate of FS-DFSP (36.3\%) is not significantly different from that of conventional DFSP; however, its rate of distant metastasis is significantly higher (13\%) compared with the conventional type (8). In addition, tumor-related mortality was observed in $5.8 \%$ of FS-DFSP cases (3). In contrast, the local recurrence rate of pigmented DFSP (10-13\%) is lower compared with conventional DFSP, and distant metastasis is extremely rare (6).

The presence of a fibrosarcomatous area in pigmented DFSP is extremely rare. Table I summarizes the clinicopathological features of the five previously reported fibrosarcomatous pigmented DFSP cases (3-7) along with the present one. This lesion mainly affects middle-aged males (average 49.2 years old, range 27-75 years; male to female ratio 5:1), and occurs mostly in the extremities. A fibrosarcomatous component was present in the primary lesion in two of the 6 cases, including the present case, and appeared in the locally recurrent or metastatic lesions in 3 cases (Table I). Melanin-containing spindle cells were present only in the conventional DFSP component in all cases.

The prognosis of this lesion appears to be poor, although the number of reported cases is limited; 4 of 6 cases displayed multiple metastases and three of the patients succumbed to the disease. The remaining case (case 3) had local recurrence, and the present case has been free from local recurrence and metastasis during 2 months of medical follow-up. We conclude that the presence of a fibrosarcomatous component in pigmented DFSP is associated with aggressive behavior; thus, careful observation and assessment for the presence of a fibrosarcomatous component is necessary in the diagnosis of pigmented DFSP.

\section{References}

1. Weyers W, Mentzel T, Kasper RC, et al: Dermatofibrosarcoma protuberans. In: World Health Organization Classification of Tumours. Pathology and Genetics of Skin Tumours. LeBoit PE, Burg G, Weedon D and Sarasain A (eds). IARC Press, Lyon, pp259-261, 2006.

2. Dupress WB, Langloss JM and Weiss SW: Pigmented dermatofibrosarcoma protuberans (Bednar tumor): A pathologic, ultrastructural, and immunohistochemical study. Am J Surg Pathol 9: 630-639, 1985.

3. Mentzel T, Beham A, Katenkamp D, Dei Tos AP and Fletcher CDM: Fibrosarcomatous ("high-grade") dermatofibrosarcoma protuberans: clinicopathologic and immunohistochemical study of a series of 41 cases with emphasis on prognostic significance. Am J Surg Pathol 22: 576-587, 1998.

4. Onoda N, Tsutsumi Y, Kakudo K, et al: Pigmented dermatofibrosarcoma protuberans (Bednar tumor). An autopsy case with systemic metastasis. Acta Pathol Jpn 40: 935-940, 1990.

5. Porter C, Vincetic A, Saleh ME and Goldstein H: Pigmented dermatofibrosarcoma protuberans of the foot with fibrosarcomatous changes: a review and case presentation. J Foot Ankle Surg 41: 186-191, 2002.

6. Suehara Y, Yazawa Y and Hitachi K: Metastatic Bednar tumor (pigmented dermatofibrosarcoma protuberans) with fibrosarcomatous change: a case report. J Orthop Sci 9: 662-665, 2004.

7. Kini H, Raghuveer CV and Pai MR: Fibrosarcomatous Bednar tumor with distant metastases - a case report. Indian J Pathol Microbiol 47: 26-29, 2004.

8. Voth H, Landsberg J, Hinz T, et al: Management of dermatofibrosarcoma protuberans with fibrosarcomatous transformation: an evidence-based review of the literature. J Eur Acad Dermatol 25: 1385-1391, 2011. 